\title{
Nutraceuticals: Frontier in Healthcare
}

\author{
Firoj A Tamboli ${ }^{1 *}$, Harinath N More ${ }^{1}$, Vishal H Thorat ${ }^{1}$, Asha S Jadhav ${ }^{1}$ \\ and Niolfar A Tamboli ${ }^{2}$ \\ ${ }^{1}$ Department of Pharmacognosy, Bharati Vidyapeeth College of Pharmacy, \\ Maharashtra, India \\ ${ }^{2}$ Hon. Shri. Annasaheb Dange Ayurved Medical College, Post Graduate and Research \\ Center, Maharashtra, India \\ *Corresponding Author: Firoj A Tamboli, Head, Department of Pharmacognosy, \\ Bharati Vidyapeeth College of Pharmacy, Maharashtra, India.
}

Received: April 09, 2021

Published: June 07, 2021

(C) All rights are reserved by Firoj A

Tamboli, et al.

\begin{abstract}
Nutraceutical is a term used for segregated products from processed foods, supplements, specialty foods and food products that are used as nutrients in balanced foods. The lifestyles of people around the world have changed over the past century due to increased incomes, declining exercise and preferences for unhealthy foods. Nutraceuticals are very popular because of their safety, nutritional value and treatment. They are used in the treatment and prevention of various diseases, including heart disease, cancer, diabetes, Alzheimer's disease, stroke and Parkinson's disease, as well as obesity. Better understanding "from treatment to prevention" leads to an increase in the demand for nutraceutical. Nutraceutical has lead to in a new era of medicine and health, which has made the food and pharmaceutical industry a business of research interest.
\end{abstract}

Keywords: Nutraceutical; Balanced Foods; Parkinson's Disease; Obesity; Antioxidants

\section{Introduction}

Hippocrates, the great Greek physician, once said, "Let food be your medicine and medicine is your food." Although food-based medicine has recently been at the forefront of wellness trends, the concept of using food as a medicine has existed for thousands of years. The term "nutraceuticals" was coined in 1989 by Stephen De Felice, founder and president of the Medical Innovation Foundation in Cranford, New Jersey. It has been defined as "a food or part of a food that provides health or health benefits including the prevention and treatment of disease" [1].

Nutrients can range from isolated nutrients, food bases, nutritional supplements and foods to genetically modified foods and processed products, including cereals, sauces and beverages. In addition, these products can have physiological functions and bio- logical effects [3]. Because rationality follows the word "prevention is better than cure", nutraceuticals are becoming a major alternative for pharmacists [4]. With the adoption of the Health and Nutrition Supplements Education Act 1994, the definition of nutraceuticals was extended to include vitamins, minerals, herbs and other plant substances, amino acids and other nutrients used to supplement human nutrition, thereby increasing overall dietary intake and consequently dramatically increasing use of nutraceuticals [5].

Nutraceuticals are constantly evolving and have spread rapidly throughout the world [6]. The global nutraceutical market is expanding at a compound annual growth rate (CAGR) of $7.5 \%$, according to a recent analysis of the BCC survey, reaching $\$ 285.0$ billion in 2021 from \$ 198.7 billion in 2016 [7-10]. 
Categories of nutraceuticals [11-16]

- Herbals -Herbs or botanical products as concentrates and extracts

- Nutrients-Substances with established nutritional functions, such as vitamins, minerals, amino acids and fatty acids

- Dietary supplements - includes non-starchy polysaccharides such as cellulose, hemicelluloses, gum and pectin, lignin and resistant dextrins.

- Phytochemicals- are polyphenols, isoflavonoids, anthocyanidins, phytoestrogens, terpenoids, carotenoids, limonoids, phytosterols, glucosinolates, and polysaccharides

- Prebiotics- A fermented dietary ingredient that allows specific changes both in the composition and/or the activity of the gastrointestinal microbiota that exchange benefits upon the host wellbeing and health.

- $\quad$ Probiotics- are live microbial feed supplement for improving its intestinal microbial balance. Lactobacillus bacteria and bifidobacteria are most important and probiotics

- Nutraceutical enzymes.

\section{Benefits of nutraceuticals}

Nutraceuticals may helpful to improve overall health, boost energy relieve anxiety improve mental clarity enhance sleep quality and quantity prevent chronic diseases reduce drug cravings delay the aging process increase life expectancy and to support and regulate bodily functions [17].

Use of nutraceuticals in preventing diseases and to promote health

In addition to these health benefits, recent studies have shown promising results in the efficacy of herbal nutraceuticals in oxidative stress disorders, including allergies, Alzheimer's disease, cardiovascular disorders, cancer, diabetes, inflammatory diseases, Parkinson's disease, and obesity.

\section{Cardio vascular disease and nutraceuticals}

Cardiovascular problems or problems that affect the heart and blood vessels are one of the biggest health problems in the world. Fiber, antioxidants, omega- 3 polyunsaturated fatty acids, vitamins, minerals for the prevention and treatment of CVS [18]. Flavonoids present in vegetables such as onions, fruits such as grapes, apples and cherries block angiotensin converting enzyme, which is con- sidered to be result in a drop in blood pressure and prevent deadly heart disease and myocardial infarction. Ginger for its antioxidant and anti-inflammatory properties, is used in the treatment of hypertension and palpitations. Green and yellow vegetables rich in phytosterols also reduce CDV by blocking cholesterol absorption [19].

\section{Cancer and nutraceuticals}

There is a huge need in clinics to prevent cancer progression through non-invasive therapeutic approaches, as all the cancer treatment options currently available are expensive, but none are safe. Nutraceuticals are used to treat chronic diseases and to improve human health. Flavonoids, which block estrogen-producing enzymes, reduce estrogen-induced cancer. A wide range of phytodrugs with alleged hormonal activity, called 'phytoestrogens', are recommended to prevent prostate/breast cancer. Soy-based foods, which are a source of isoflavones, curcumin curry and soy isoflavones, have chemopreventive properties against cancer. Lycopene is concentrated in the skin, testicles, adrenal glands and prostate, where it protects against cancer [20-23]. Some promising natural compounds that have shown excellent results in vitro and in vivo are: chebulagic acid, apigenin, norcantharidine, saffron/crocin, partenolide, longicaurin E, lupup, spongistatin 1 and deoxyvariol B [24].

\section{Obesity and nutraceuticals}

Obesity is a global public health problem and is defined as the accumulation of unwanted amounts of body fat [25]. Herbal stimulants such as ephedrine. caffeine, ma huang-guarana, chitosan and green tea help with weight loss [26]. Buckwheat seed proteins act similarly to the fibers in foods. 5-Hydroxytryptophan and green tea extract may promote weight loss, while the former reduces appetite and the latter increases energy expenditure [27]. The mixture of glucomannan, chitosan, hay and vitamin $\mathrm{C}$ in a dietary supplement significantly reduced body weight [28].

\section{Osteoarthritis and nutraceuticals and nutraceuticals}

Nutraceuticals and C-phycocyanin (CPC) components may be able to mediate the three primary pathogenic mechanisms of osteoarthritis: inflammation, Chondral degeneration, and oxidative stress in vitro [29]. Glucosamine (GLN) and chondroitin sulfate (CS) are widely used to alleviate the symptoms of OA. These nutraceu- 
ticals have nutritional and pharmaceutical properties and appear to regulate the expression and synthesis of NO and PGE2 genes and provide a credible explanation for their anti-inflammatory activities [30,31]. Methylsulfonylmethane (MSM) and boswellic acid (BA) have been shown to be effective supplements in the treatment of inflammation and joint degeneration [32].

\section{Anti-inflammatory activities and Nutraceuticals}

Quercetin, flavones/flavonols, kemperol, myricetin, fisetin were reported to possess LO and COX inhibitory effects [33,34]. Cucurmin, which is a polyphenol in turmeric, has anti-cancer, antioxidant and anti-inflammatory properties [35]. Linoleic acid is used to treat inflammatory problems and autoimmune diseases [36].

\section{Antioxidant properties and nutraceuticals}

Special groups of substances, such as flavonoids, vitamins, minerals, and carotenoids, have antioxidant properties. These substances help stimulate the immune and digestive systems and modulate inflammatory and degenerative processes in the body [37]. According to a recent study, dietary supplementation with antioxidants can help prevent skin cancer [38]. The Alliaceae family contains specific sulfur compounds, Brassicaceae contains glucosinolates, and the Lamiaceae herbs contain rosemary acid, an important bioactivity with antioxidant and anti-inflammatory effects [39].

\section{Alzheimer's disease and nutraceuticals}

Lutein, lycopene, turmerin, $\beta$-carotene, curcumin, etc. they can have positive effects on specific diseases and neutralize the negative effects of oxidative stress, mitochondrial dysfunction and various forms of nerve degeneration [40]. Several studies report an association between higher food antioxidant intake and reduced risk in patients with Alzheimer's disease, which is very necessary because disease prevention is significantly cooler than treatment [41]. Consumption of foods rich in polyunsaturated fatty acids and saturated and trans-fatty acids tends to suppress neurodegeneration, while foods rich in trans fats can increase neurodegeneration [42].

\section{Parkinson's disease and nutraceuticals}

Vitamin E in food can protect against Parkinson's disease. Canadian researchers have suggested that vitamin $\mathrm{E}$ in food may protect against Parkinson's disease. Creatine appears to have modified the characteristics of Parkinson's disease, as measured by a decrease in clinical symptoms [43]. Various vitamins can be used in Parkinson's disease, including vitamin B3, vitamin B9 or folic acid, vitamin B12, vitamin B6, vitamin D, vitamin E and vitamin C.

\section{Allergies and nutraceuticals}

Allergy is a disorder of the hypersensitivity of the immune system. Allergic reactions are remarkable due to overactivation of mast cells and basophils. Quercetin, an antioxidant, eliminates free radicals and treats allergies [45]. When an individual comes in contact with an allergen, their immune system responds by producing histamines or chemicals that cause allergy symptoms, such as nasal congestion or watery eyes. To address adverse side effects, many pharmaceutical companies have developed a natural antihistamine called Histamine Shield. This supplement stimulates the body's immune response, so the allergic reaction can be alleviated.

\section{Diabetes and nutraceuticals}

Globally, type 2 diabetes is one of the main issues. We can facilitate this with the application of nutrition. The omega-3 is very popular, the omega- 6 found in fish, reducing the glucose tolerance. The isoflavones are phytoestrogens which are structurally associated with human estrogen, associated with the lower incidents and mortality rate of type II diabetes mortality [46].

\section{Conclusion}

Traditional products have been known for their healing properties for centuries. These plants can be a better choice than their nutritional building capacity. Nutraceuticals that have demonstrated their health benefits and potential to prevent disease, should be taken in accordance with the accepted recommended. In today's pharmaceutical perspective, healthy eating plays an important role in therapeutic development. However, its success depends on ensuring its quality, purity, safety and effectiveness. Nutraceuticals are widely used in all age groups because of their safety, high content, purity and effectiveness. In this new age, therefore, we must say, "Let food be your medicine, and the use of good things can prevent you from doing so". 


\section{Bibliography}

1. De Felice SL. "The nutraceutical revolution, its impact on food industry". Trends in Food Science and Technology 6.2 (1995): 59-61.

2. Mannion M. "Nutraceutical revolution continues at Foundation for Innovation in Medicine Conference". American Journal of Natural Medicines 5 (1998): 30-33.

3. Andlauer W and Furst P. "Nutraceuticals: a piece of history, present status and outlook". Food Research International 35 (2002): 171-176.

4. Ruchi S., et al. "Role of Nutraceuticals in health care: A review". International Journal of Green Pharmacy 11 (2017): 386-394.

5. Whitman M. "Understanding the perceived need for complementary and alternative nutraceuticals: lifestyle issues". Clinical Journal of Oncology Nursing 5 (2001): 190-194.

6. Childs NM. "Nutraceutical industry trends". Nutraceuticals: Nutritionally Functional Foods 2.1 (1999): 73-85.

7. Golla U. "Emergence of nutraceuticals as the alternative medications for pharmaceuticals". International Journal of Complementary and Alternative Medicine 11.3 (2018): 155-158.

8. Brower B. "Nutraceuticals: Poised for a healthy slice of the market”. Nature Biotechnology 16.8 (1998): 728-731.

9. https://www.bccresearch.com/market-research/food-andbeverage/nutraceuticals-markets-report-fod013f.html

10. De Felice SL. "Nutraceuticals: Opportunities in an Emerging Market". Script Magazine (1992).

11. Dureja H. "Developments in nutraceuticals". Indian Journal of Pharmacology 35 (2003): 363-372.

12. Mobarhan S., et al. "Micronutrient supplementation trials and the reduction of cancer and cerebrovascular incidences and mortality". Nutrition Review 52 (1994): 102-105.

13. Jong WP. Anesthesia and Analgesia 97 (2003): 748.
14. Braeckman J. "The Extract of Serenoa repens in the treatment of benign prostatic hyperplasia: a multicenter open study". Current Therapeutic Research 55 (1994): 776-785.

15. Craig W and Beck L. "Phytochemicals: Health Protective Effects". Canadian Journal of Dietetic Practice and Research 60 (1999): 78-84.

16. Klein C., et al. "From food to nutritional support to specific nutraceuticals: a journey across time in the treatment of disease". Journal of Gastroenterology 35 (2000): 1-6.

17. Pandey M., et al. "Nutraceuticals: New era of medicine and health". Asian Journal of Pharmaceutical and Clinical Research 3-1 (2010): 11-15.

18. Garima Verma., et al. International Journal of Pharmacy and Therapeutics 7-4 (2016): 152-160.

19. Thomasset SC., et al. "Dietary polyphenolic Phytochemicalspromising cancer chemopreventive agents in humans? A review of their clinical properties". International Journal of Cancer 120 (2007): 451-458.

20. Frydoonfar HR., et al. "The variable effect on proliferation of a colon cancer cell line by the citrus fruit flavonoid Naringenin". Colorectal Disease 5 (2003): 149-152.

21. Limer JL and Speirs V. "Phyto-oestrogens and breast cancer chemoprevention". Breast Cancer Research 6 (2004): 119-127.

22. Mandel S., et al. "Proceedings from the Third Int. Conf. Mechanism of Action of Nutraceuticals". Journal of Nutritional Biochemistry 16 (2005): 513-520.

23. Kucuk 0., et al. "Lycopene in the Treatment of Prostate Cancer". Pure and Applied Chemistry 74 (2002): 1443-1450.

24. Ferrucci V., et al. "Natural compounds for pediatric cancer treatment". Naunyn-Schmiedeberg's Archives of Pharmacology 389 (2016): 131-149.

25. Caterson ID and Gill TP. "Obesity: epidemiology and possible prevention". Best Practice and Research: Clinical Endocrinology and Metabolism 16.4 (2002): 595-610. 
26. Si-quan L and Zhang Q H. "Advances in the development of functional foods from buckwheat". Critical Review in Food Science and Nutrition 41 (2001): 451-464.

27. Bell SJ and Good rick G K. "A Functional Food Product for the Management of Weight". Critical Review in Food Science and Nutrition 42 (2002): 163-178.

28. Woodgate DE and Conquer JA. "Prevalence of self-treatment with complementary products and therapies for weight loss: A randomized, cross-sectional Study in Overweight Obese Patients in Colombia". Current Therapeutic Research 64 (2003): 248-262.

29. Martinez SE., et al. " Pharmacological effects of a C-phycocyanin-based multicomponent nutraceutical in an in-vitro canine chondrocyte model of osteoarthritis". Canadian Journal of Veterinary Research 79 (2015): 241-249.

30. Bottegoni C., et al. "Oral chondroprotection with nutraceuticals made of chondroitin sulphate plus glucosamine sulphate in osteoarthritis". Carbohydrate Polymer 109 (2014): 126-138.

31. Kalioraa A C., et al. "Dietary antioxidants in preventing atherogenesis". Atherosclerosis 187 (2006): 1-17.

32. Notarnicola A., et al. "Methylsulfonylmethane and boswellic acids versus glucosamine sulfate in the treatment of knee arthritis: Randomized trial". International Journal of Immunopathology and Pharmacology 29 (2015): 140-146.

33. Kim HP., et al. "Effects of naturally occurring flavonoids and bioflavonoids on epidermal cycloxygenase and lipoxygenase from guinea pigs". Prostaglandins, Leukotrienes and Essential Fatty Acids 58 (1998): 17-24.

34. Jachak SM. "Natural products: Potential source of COX inhibitors”. CRIPS 2.1 (2001): 12-15.

35. Srivastava Shubhra. "Nutraceuticals: A Review". Journal of Chronotherapy and Drug Delivery 6.1 (2015): 1-10.

36. Formica J V and Regelson W. "Review of the Biology of Quercetin and Related Bioflavonoids". Food and Chemical Toxicology 33 (1995): 1061-1080.

37. VranesiA Bender D. "The role of nutraceuticals in anti-aging medicine". Acta Clinica Croatica 49 (2010): 537-544.
38. Katta R and Brown DN. "Diet and Skin Cancer: The Potential Role of Dietary Antioxidants”. Journal of Skin Cancer 7 (2015): $1-10$.

39. Shan B., et al. "Antioxidant capacity of Tewnty Six spice extracts and characterization of their phenolic constituents". Journal of Agricultural and Food Chemistry 20.53 (2005): 7749-7759.

40. Glenville M. "Nutritional supplements in pregnancy: commercial push or evidence based". Current Opinion in Obstetrics and Gynecology 18 (2006): 642-647.

41. Nunomura A., et al. "Oxidative Damage is the Earliest Event in Alzheimer Disease". Journal of Neuropathology and Experimental Neurology 60 (2001): 759-767.

42. Dohrmann DD., et al. "Japanese, Mediterranean and Argentinean Diets and Their Potential Roles in Neurodegenerative Diseases". Food Research International 120 (2019): 464-477.

43. Brower V. "A nutraceutical a day may keep the doctor away". EMBO Reports 6.8 (2005) 708-711.

44. Tatton, W. et al. “Apoptosis in Parkinson's disease: Signals for neuronal degradation". Annals of Neurology 53.3 (2003): S61S70.

45. Ramaa CS., et al. "Nutraceuticals- An emerging era in the treatment and prevention of cardiovascular diseases". Current Pharmaceutical Biotechnology 7 (2006): 15-23.

46. Chauhan B., et al. "Current Concepts and prospects of herbal Nutraceuticals: A Review". Journal of Advanced Pharmaceutical Technology and Research 4 (2013): 4-8.

\section{Volume 5 Issue 7 July 2021 (C) All rights are reserved by Firoj A Tamboli., et al.}

Citation: Firoj A Tamboli., et al. "Nutraceuticals: Frontier in Healthcare". Acta Scientific Nutritional Health 5.7 (2021): 22-26. 